\title{
La lipase hormono-sensible : que nous apprend l'invalidation du gène?
}

La lipase hormono-sensible (LHS) catalyse l'hydrolyse des triglycérides, des esters de cholestérol et des esters de rétinol. Son rôle et les mécanismes de sa régulation ont été particulièrement étudiés dans les adipocytes du tissu adipeux blanc qui accumulent, sous forme de triglycérides, la plus grande partie des réserves énergétiques de l'organisme [1]. La stimulation par les catécholamines des récepteurs $\beta$-adrénergiques active, via l'augmentation des concentrations intracellulaires d'AMP cyclique, la protéine kinase A qui induit la phosphorylation de la LHS sur plusieurs résidus sérinc et entraîne une forte induction de son activité lipolytique [2]. L'étape limitante de la lipolyse adipocytaire est l'hydrolyse des triglycérides en diglycérides par la LHS phosphorylée. L'activation de l'enzyme s'explique en partie par une translocation du compartiment cytosolique vers la gouttelette lipidique, mais pourrait également faire intervenir des changements de conformation qui conduiraient à démasquer le site actif au contact du substrat. Le rôle de la L.HS dans les autres tissus a été en revanche beaucoup moins étudié. Dans le tissu adipeux brun, les acides gras libérés lors de la lipolyse par la L.HS sont des activateurs de la protéine découplante UCPI (uncoupling protein) (m/s 1997, $\left.n^{\circ} 8-9, p .1061\right)$, et donc de la réponse thermogénique de ce tissu. Dans le muscle squelettique, l'enzyme serait impliquée dans la mobilisation des triglycérides, en particulier dans les fibres à métabolisme oxydatif [3]. La L.HS est également exprimée dans les cellules $\beta$ du pancréas [4] où la lipolyse endogène
L'activité d'hydrolyse des esters de cholestérol de la LHS pourrait jouer un rôle dans la stéroïdogenèse au niveau des glandes surrénales et du corps jaune, et dans la prévention de la formation des cellules spumeuses à partir des macrophages $[5,6]$. Enfin, dans les testicules, une forte expression de la I.HS a été observée dans les cellules germinales mâles [7]. La transcription de cette forme testiculaire est sous le contrôle d'un promoteur distinct du promoteur de la forme adipocytaire $[8,9]$, et la protéine testiculaire possède un domaine amino-terminal spécifique, codé par un exon transcrit uniquement dans le testicule [7].

Osuga et al. viennent de décrire les premières données obtenues chez des souris dont le gène de la LHS a été invalidé (souris $L H S^{-/-}$) [10]. L'expression de la LHS et l'activité invitro d'hydrolyse des esters de cholestérol sont totalement abolies dans les tissus adipeux et le testicule. Le phénotype le plus marquant est la stérilité des souris mâles alors que la fertilité des femelles est préservée. Les testicules des souris $\mathrm{IH} \mathrm{S}^{-1-}$ sont plus petits et on observe une diminution drastique du nombre de spermatozoïdes dans l'épididyme, les spermatozoïdes résiduels n'étant pas mobiles. L'examen histologique des testicules révèle une diminution de l'épaisseur des tubes séminifères et du nombre de spermatides mûrs, suggérant un blocage de la spermatogenèse. Plusieurs éléments indiquent que la stérilité des souris mâles n'est pas due à une insuffisance gonadique : les animaux s'accouplent en effet normalement et les concentrations plasmatiques de testostérone, d'hormone lutéinisante et d'hor- mone folliculo-stimulante sont normales. Si le contenu des testicules en triglycérides est normal, la quantité d'esters de cholestérol est en revanche le double de celle d'animaux normaux. ('es données suggèrent que la LHS, via l'hydrolyse des esters de cholestérol et peut-être d'autres substrats, joue un rôle direct dans la spermatogenèse. L.étude biochimique de la forme testiculaire de la I.HS, avec son domaine amino-terminal spécifique, pourrait révéler l'existence de substrats préférentiels différents de ceux de la forme adipocytaire. L'importance des lipides testiculaires dans la spermatogenèse est encore peu connue. Un déficit en acides gras essentiels conduit à une dégénérescence testiculaire [11]. Une autre possibilité serait une implication de la L.HS testiculaire dans le métabolisme des esters de rétinol. On sait en effet qu'un déficit en vitamine $A$ ou que l'inactivation des gènes codant pour des récepteurs des rétinoïdes RAR $\alpha$ et RXR $\beta$ conduit à une spermatogenèse anormale et à la stérilité des animaux [12, 13]. Il sera intéressant de déterminer si la LHS contrôle la production de l'acide rétinoïque, ligand de ces récepteurs. L'identification précise des cellules germinales qui l'expriment facilitera aussi l'analyse de son rôle exact. Une autre question soulevée par cette étude concerne la régulation de l'activité de la LHS dans les cellules germinales. Cette enzyme estelle activée, comme l'est la forme adipocytaire, par phosphorylation et, dans ce cas, quels sont les récepteurs membranaires et la voie de transduction impliqués? Chez l'homme, on peut envisager que certaines stérilités soient associées à des altérations de 
l'expression de la L.HS testiculaire même si, contrairement aux rongeurs, deux transcrits de la LHS sont exprimés [7].

Les conséquences de l'absence d'expression de la L.HS dans les tissus adipeux brun et blanc sont nettement moins importantes qu'elles ne le sont dans le testicule. L'activité d'hydrolyse invitro des triglycérides ne semble pas modifiée dans le tissu adipeux brun, et la réponse thermogénique des animaux est préservée. C'es données suggèrent l'existence d'une autre enzyme responsable de l'hydrolyse des triglycérides dont l'activité serait aussi réglée, soit directement par les catécholamines, par phosphorylation, soit indirectement par induction de son expression. L'absence de LHS entraine cependant une dérégulation du métabolisme lipidique dans le tissu adipeux brun chez les souris $L H S^{2 /-}$. L'hypertrophie des adipocytes bruns s'accompagne d'une augmentation du poids du tissu et de la quantité de triglycérides. Dans le tissu adipeux blanc, si l'activité lipase et la stimulation de la lipolyse in vitro et in vivo par les catécholamines sont fortement diminuées, il subsiste une activité résiduelle. Il semble donc que, comme dans le tissu adipeux brun, une autre lipase soit présente. Son identité n'est pas connue mais l'implication de la lipase des monoglycérides ou de celle des lipoprotéines semble exclue. Un autre aspect intéressant du phénotype des souris $L H S^{-/}$est leur absence d'obésité : en effet, la masse des différents dépôts de tissu adipeux blanc n'est pas augmentée. (iependant, la taille des adipocytes est doublée et les auteurs formulent l'hypothèse selon laquelle certains adipocyte meurent en raison d'une accumulation exagérée de lipides.
L'hypertrophie des adipocytes blancs et la réduction marquée de la lipolyse stimulée par les catécholamines permettent d'entrevoir des perspectives intéressantes dans l'utilisation de ce modèle. D'une part, la sécrétion de leptine étant corrélée positivement à la taille cellulaire, la prise alimentaire des souris $L H S^{2 /-}$ pourrait être modifiée. D'autre part, les concentrations plus basses d'acides gras libres plasmatiques pourraient avoir un impact sur leur utilisation par le muscle squelettique et affecter le métabolisme du glucose dans ce tissu. Ces altérations conduisent à s'interroger également sur le statut insulinique des animaux : en effet, les petits adipocytes étant plus sensibles à l'action de l'hormone [14], on peut envisager l'existence d'une résistance à l'action de l'insuline dans les adipocytes hypertrophiés des souris $L H S^{-1-}$. En revanche, une meilleure sensibilité à l'action de l'insuline pourrait être observée dans le muscle squelettique et le foie du fait de la diminution des concentrations circulantes d'acides gras libres. L'étude d'Osuga et al. n'aborde pratiquement pas le rôle de la LHS dans les autres tissus. L'activité d'hydrolyse des esters de cholestérol n'est pas modifiée dans les macrophages péritonéaux, ce qui suggère l'implication d'une autre estérase. Il reste à déterminer les effets de l'absence de L.HS sur la sécrétion de l'insuline dans les cellules $\beta$ du pancréas, et sur le métabolisme du cholestérol dans les ovaires et les glandes surrénales.

1. Langin D, Holın C, Lafontan M. Aclipocyte hormone-sensitive lipase: a ma jor regulator of lipic metabolism. Proc Nutr Soc 1996; 55: 93-109. 2. Lafontan M, Langin D. Régulation neurohumorale de la lipolyse: aspects physiologiques et pathologiques. Med Sici 1998; 8-9: 865-76. 3. Langfort J, Ploug T, lhlemann J, Salclo M,
Holm C, Galbo H. Expression of hormone-sensitive lipase and its regulation by adrenaline in skeletal muscle. Biochem / 1999; 340 : 459-65.

4. Mulder H, Stenson Holst L, Svensson H, et al. Hormone-sensitive lipase, the rate-limiting enzyme in triglyceride hydrolysis, is expressed and active in $\beta$-cells. Diabetes 1999; 48: 228-32.

5. Yeaman SJ. Hormone-sensitive lipase: a multipurpose enzyme in lipid metabolism. Biochim Biophys Acta 1990; 1052 : 128-32.

6. Escary JL, Choy HA, Reue K, Schotz MC. Hormone-sensitive lipase overexpression increases cholesteryl ester hycholysis in macrophage foam cells. Aiterioscler Thromb Vasc Biol 1998; 18:991-8. 7. Stenson Holst L, Langin D, Mulder H, et al. Molecular cloning, genomic organization, and expression of a testicular isof orm of hormone-sensitive lipase. (ienomics 1996; 35: 441-7.

8. Grober J, Laurell H, Blaise R, et al. Characterization of the promoter of human adlipocyte hormone-sensitive lipase. Biochem / 1997; 328: 453-61. 9. Blaise R, Grober J, Rouet P, Tavemier G, Daegelen D, Langin D. Testis expression of hormonesensitive lipase is conf erred by a specific promoter that contains four regions binding testicular nuclear proteins. / Biol Chem 1999; 274: 9327-34. 10. Osuga J1, lshibashi S, Oka T, et al. Targeted clisruption of hormone-sensitive lipase results in male sterility and adlipocyte hypertrophy, but not in obesity. Proc Nall Acad Sci (ISA 2000; 97 : 787-92. 1 1. MacDonald ML, Rogers QR, Morris J(;, Ciupps PT. Effects of linoleate and arachidonate deficiencies on reproduction and spermatogenesis in the cat. / Nutr 1984; 114 : 719-26.

12. Luikin ' $\Gamma$, Lohnes D, Mark M, et al. High posthatal lethality and testis degeneration in retimoic acid receptor a mutant mice. Proc Natl Acad Sci USA 1993; 90 : 7225-9

13. Kastner P, Mark M, Leid M, et al. Abnormal spermatogenesis in RXR mutant mice. (ienes bev $1996 ; 10: 80-92$

14. Okimo A, Tamemoto H, Tobe K, et al. Troglitazone increases the number of small adipocytes without the change of white adipose tissue mass in obese Zucker rats. J C.lin Invest 1998; 101 : 135461.

\section{Dominique Langin Stéphanie Lucas Régis Blaise}

Inserm U317, Institut Louis-Bugnard, CHU Rangueil, 31403 Toulouse Cedex 4, France.

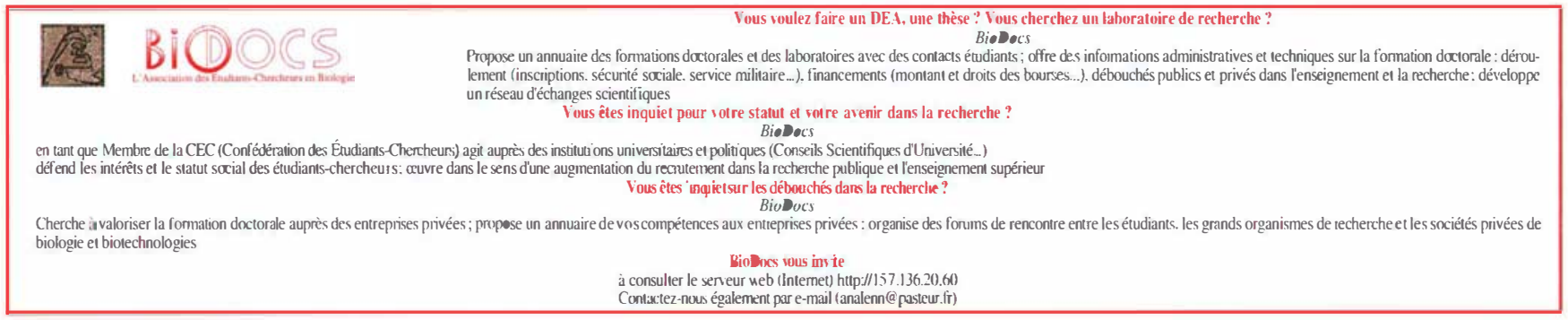


Les protéases font l'ouf. L'ovulation est un processus parfaitement réglé dans le temps, au cours duquel l'ovocyte mûr est libéré du follicule ovarien. Cet événement est précédé d'un pic de synthèse de la LH (luteinizing hormone), directement responsable de l'induction de l'expression du récepteur nucléaire de la progestérone (PR) dans les cellules de la granulosa. Or, cette étape semble indispensable à l'ovulation puisque les souris femelles déficientes pour ce récepteur $\left(P R^{-1-}\right)$ sont stériles, en raison précisément d'un défaut d'ovulation [1]. La première étape nécessaire à la libération de l'ovule est la dégradation de la paroi du follicule, et il était donc logique de penser que les gènes sous le contrôle du PR pourraient être impliqués dans ce phénomène. Par ailleurs, de nombreux travaux suggéraient l'intervention de protéases dans ce remodelage tissulaire. En utilisant les souris $P R^{-1-}$, l'équipe de J.S. Richard, à Houston, a mis en évidence le rôle de protéines candidates dans le contrôle de l'ovulation [2]. Deux d'entre elles sont clairement induites en réponse à la $\mathrm{L} . \mathrm{H}$ chez les souris sauvages mais pas chez les $P R^{-/-}$: il s'agit d'ADAMTS-1 $\left(\mathrm{m} / \mathrm{s} 1999, n^{\circ} 1, p .117\right.$ et $n^{\circ} 10$, p. 1148) (A disintegrin and metalloproteinase with thrombospondin-like motifs) et de la cathepsine-I, une sérine protéase lysosomale. I.e profil d'expression spatio-temporelle de ces deux protéines concorde avec leur dépendance au PR. I.es substrats de la cathepsine $\mathrm{L}$ incluent les collagènes (type l et IV), l'élastine et la fibronectine, tous susceptibles de maintenir l'intégrité de la paroi folliculaire. La protéine ADAMTS-l possède plusieurs fonctions, l'une de protéase, l'autre de facteur angiostatique qui pourrait participer également au contrôle de l'inflammation et de la néovascularisation intervenant lors de la forma- tion du corps jaune. Il reste à déterminer si les effets du PR sur les gènes de ADAMTS-1 et de la cathepsine-L sont directs ou indirects ainsi que les mécanismes transcriptionnels impliqués. Cette étude a aussi permis d'écarter l'hypothèse d'un rôle de certaines MMP (matrix metalloproteinases) dans l'ovulation. En effet, l'analyse de l'expression des MMP-2 et -9 chez les souris $P R^{-1-}$ indique que ces protéines ne sont pas impliquées dans le remodelage de la paroi folliculaire. Quant à MMP-13, son rôle demeure probable mais ne ferait pas intervenir le PR.

[1. Lydon JP, et al. Genes Dev 1995; 9: 2266-78.]

[2. Robker RL, et al. Proc Natl Acad Sci USA 2000; 97 : 4689-94.]

La télomérase : un nouveau traitement de la cirrhose hépatique? La cirrhose hépatique, qui atteint plusieurs centaines de millions de personnes dans le monde, est caractérisée par l'arrêt de la prolifération des hépatocytes et le remplacement du tissu hépatique par une fibrose progressive associée notamment à la conversion des cellules dites étoilées du foie en cellules myofibroblastiques. La physiopathogénie de cette affection et plus particulièrement les causes de l'arrêt de la prolifération sont mal connues: interactions altérées des hépatocytes avec la matrice extracellulaire, surexpression anormale de TGF $\beta 1 A$, raccourcissement anormal des télomères. Afin d'étayer cette dernière hypothèse, une équipe américaine [1] a testé le rôle du raccourcissement des télomères dans le développement des atteintes chroniques du foie induites par différents facteurs. Trois conditions pathologiques différentes ont été testées: génétique, par l'expression hépatotoxique de l'activateur du plasminogène; chirurgicale, au travers de l'hépatectomie partielle; et enfin chimique, par l'injection de tétrachlorure de carbone (CCl4). Leurs effets respectifs ont été testés dans deux groupes de souris, un groupe de souris sauvages et un groupe de souris invalidées pour le gène de la télomérase $\left(T R^{-1}\right)$. Ces dernières présentent au cours des générations un raccourcissement progressif de leurs télomères qui a déjà été impliqué dans l'altération de la prolifération ou de l'apoptose de certains tissus à renouvellement rapide comme l'intestin ou la moelle osseuse ([2] et $\left.\mathrm{m} / \mathrm{s} 1999, n^{\circ} 11, p .1286-91\right)$. Dans les trois conditions évoquées, une diminution et/ou un retard de la prolifération des hépatocytes ont été constatés chez la souris mutante $T R^{-1}$. L'injection de CCl4 constituant le modèle murin le plus fidèle de la cirrhose hépatique humaine, c'est dans cette dernière condition que l'effet de la restauration d'une activité télomérasique a été testée. Sur des critères biochimiques et histologiques, il a pu être établi que l'injection intraveineuse d'un adénovirus codant pour la télomérase permettait de recouvrer une prolifération et une fonction hépatocytaire peu différentes de la normale.

Ces observations semblent donc démontrer l'implication du raccourcissement des télomères dans la cirrhose hépatique. Sur la base de ces résultats, les auteurs vont jusqu'à proposer d'utiliser la télomérase comme moyen thérapeutique chez des patients atteints de cirrhose hépatique terminale en attente de transplantation, tout en soulignant que cette approche ne serait pas dénuée de risque oncogénique...

[1. Rudolph KL, et al. Science 2000; 287: 1253-8.]

[2. Ancelin K, et al. Med Sci 2000; 16: 481-6.] 


\section{BRÈVES}

Encore une kinase sur la voie NFKB ! La signalisation intracellulaire déclenchée par les récepteurs des cytokines de la famille du TNF est toujours un équilibre entre apoptose, résultant de l'activation des caspases, et survie cellulaire, résultant de la translocation au noyau du facteur de transcription NFкB. La libération-activation de $\mathrm{NF} \kappa \mathrm{B}$ nécessite la phosphorylation de son inhibiteur IкB sur les sérines 32 et 36 , phosphorylation essentielle à l'ubiquitination de $\mathrm{I} \kappa \mathrm{B}$ et à sa dégradation, permettant ainsi de

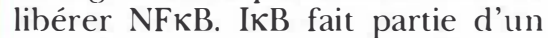
complexe comprenant les kinases IKK $\alpha$ et IKK $\beta$ (I $\kappa B$ kinase), induisant la phosphorylation de $I \kappa B$, ainsi que les molécules associées IKK $\gamma$, IKK-i et NEMO $\left(m / s ~ 1999, n^{\circ} 3\right.$, p. 419). Jusqu'ici, seule la kinase NIK (NFKB-inducing kinase) était connue pour activer ce complexe. Le groupe de Nakanishi décrit aujourd'hui une nouvelle kinase, NAK (NFKB-activating kinase) [1], qui présente une forte homologie avec les kinases IKK déjà décrites. De fait, elle est capable de phosphoryler IкB en des sites différents des sérines 32 et 36 , et son rôle principal semble plutôt de phosphoryler les deux IKK, induisant ainsi leur activation. Cet article va beaucoup plus loin en démontrant d'abord que NAK ne fait pas partie du complexe IKK et pourrait donc être le chaînon manquant entre l'activation des récepteurs et celle du complexe IKK. En effet, NAK, aussi nommée TBK1 (TANK-binding kinase), peut s'associer à TRAF2 et TANK, deux protéines associées au récepteur [2]. De plus, les auteurs démontrent que NAK est à son tour spécifiquement activée par la $\mathrm{PKC} \varepsilon$, ajoutant une nouvelle pièce au puzzle du mécanisme anti-apoptotique de l'activation des PKC.

[1. Tojima Y, et al. Nature 2000; 404: 778-82.]

[2. Pomerantz JL, et al. EMBOJ 1999 ; 18 : 6694-704.]
Une nouvelle cible des prostaglandines: la I $\kappa \mathbf{B}$ kinase. Les prostaglandines (PG) constituent une famille de médiateurs lipidiques impliqués dans la réaction inflammatoire. La signalisation des prostaglandines pro-inflammatoires est à présent bien connue. Certaines prostaglandines comme la 15dPGJ2 auraient une action anti-inflammatoire et joueraient un rôle dans la fin de la réaction inflammatoire. Ces PG comprennent un groupement cyclopentenone caractéristique. Leur mécanisme d'action suspecté était l'activation des récepteurs PPAR $\gamma$ (peroxisome proliferator activated receptor) [1]. Une étude récente du groupe de M.G. Santoro suggère un nouveau mode d'action [2]. En effet, ces PG sont capables d'inhiber l'activation de NFKB par les cytokines inflammatoires. Divers arguments ont permis de montrer que l'activité de $I \kappa B$ kinase était inhibée par ces PG. Ainsi, la phosphorylation et la dégradation de I $\mathrm{B} B$ (l'inhibiteur de $\mathrm{NF \kappa B}$ ) sont inhibées, ce qui maintient NFKB à l'état inactif cytoplasmique. Le mécanisme d'inhibition de I $\mathrm{I} B$ kinase est particulièrement original. Il s'agit d'une liaison covalente qui s'établirait entre une des cystéines du site actif de IКB kinase et le groupement cyclopentenone de la 15dPGJ2. Une IкB kinase dans laquelle cette cystéine est mutée demeure active mais n'est plus inhibée par la 15dPGJ2. Ces effets de certaines PG ne sont obtenus qu'à des concentrations micromolaires très supérieures aux concentrations des PG circulantes, mais qui peuvent être atteintes localement au cours d'un phénomène inflammatoire. Ils mettent à nouveau l'accent sur le complexe d'activation de $\mathrm{NF \kappa B}$ qui semble être une cible privilégiée de molécules anti-inflammatoires comme l'aspirine et les glucocorticoïdes.

[1. Forman BM, et al. Cell 1995; 83: 803-12.]

[2. Rossi A, et al. Nature 2000; 403: 103-8.]
ARES-SERONO FOUNDATION

WORKSHOP: «MOLECULAR

Genetics of Human Reproduc-

TION»

\section{5-17 septembre 2000}

Crète

Renseignements:

http://www.med.uoc.gr/ seroncon

\section{CONGRES DE LA SOCIÉTÉ}

FrançaISE DE Chimie

18-22 septembre 2000

Université Rennes 1

Renseignements:
Secrétariat SFC 2000
Congrès Scientifique Services (c2s)
Chantal IANNARELLI
2, rue des Villarmains, BP I24
92210 Saint-Cloud
Fax:01 47719005
E-mail: c2s@club-internet.fr
http://www.sfc.fr

5TH INTERNATIONAL MEETING

ON MOLECULAR EPIDEMIOLOGY AND EVOLUTIONARY GENETICS IN INFECTIONS DISEASES

\section{2-16 novembre 2000 \\ Hyderabad, Inde}

Renseignements:

Centre d'Études sur le Polymorphisme des Micro-organismes IRD, Montpellier, France E-mail : michel.Tibayrenc@cepm.mpl.orstom.fr 
Les co-activateurs des récepteurs nucléaires prennent du galon. L'activité transcriptionnelle des récepteurs nucléaires $(\mathrm{m} / \mathrm{s} 1998$, $n^{\circ} 11, p .1211$ et $p .1217 ; 1999, n^{\circ} 1$, p.52) dépend du ligand, mais est également influencée par divers coactivateurs dont l'importance commence à être révélée. Bien que se liant à des séquences d'ADN très similaires, les récepteurs nucléaires exercent des fonctions spécifiques. Un bon exemple est donné par les isoformes des PPAR (Peroxisomes proliferator activated receptors). $\operatorname{PPAR} \alpha$ induit les enzymes de la $\beta$-oxidation des acides gras et PPAR $\gamma$, la différenciation adipocytaire $(\mathrm{m} / \mathrm{s} 2000$, $\left.n^{\circ} 2, p .253\right)$. Quant à PPAR $\delta$, ses fonctions sont moins bien connues, sauf que l'on sait qu'il n'exerce pas d'action adipogénique [1]. La différence fonctionnelle entre PPAR $\alpha$ et $\operatorname{PPAR} \gamma$ a été astucieusement utilisée pour cloner un co-activateur spécifique de PPAR $\gamma$ [2]. A l'aide de protéines chimères PPAR $\gamma / \mathrm{PPAR} \delta$, la séquence de PPAR $\gamma$ lui conférant sa capacité adipogénique a été déterminée puis utilisée comme appât dans le crible d'une banque préparée à partir d'adipocytes. La protéine obtenue, PGC-2 (PPAR gamma coactivator-2), active sélectivement l'activité transcriptionnelle de PPAR $\gamma$ et stimule la différenciation adipocytaire. Pourquoi PGC-2 ? Parce que le même groupe a aussi cloné PGC-1, autre co-activateur de PPAR $\gamma$ qui exerce un rôle complètement différent puisqu'il stimule l'expression de gènes impliqués dans la production de chaleur et la biogenèse mitochondriale $[3,4]$. PGC-1 est fortement induit par le froid dans le tissu adipeux brun, ce qui suggère qu'il représente le lien entre ce stimulus et la réalisation d'un programme adaptatif dans ce tissu. La présence de l'un ou l'autre de ces co-activateurs dans un tissu donné, en coordination avec celle de ligands endogènes, ou exogènes, entraine donc PPAR $\gamma$ dans deux voies distinctes: différenciation adipocytaire pour PGC-2 et thermogenèse adaptative pour PGC-1. La question se pose alors de savoir si l'on pourra un jour influencer l'activité ou l'expression des co-activateurs et, par ce biais, contrôler le déroulement de certaines fonctions cellulaires normales ou pathologiques.

[1. Bastie C, et al. J Biol Chem 1999; 274: 21920-5.]

[2. Castillo G, et al. EMBO / 1999 ; $18: 3676-87$.

[3. Puigserver P, et al. Cell 1998; 92 : 829-39.]

[4. Wu Z, et al. Cell 1999; 98: 11524.]

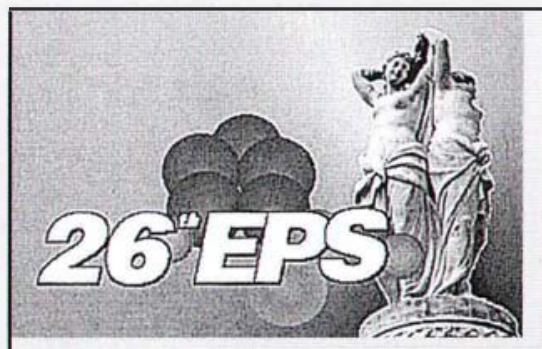

\title{
26. SYMPOSIUM EUROPEEN DES PEPTIDES
}

\author{
Montpellier, France \\ 10-15 septembre 2000
}

- Le 26e Symposium Européen des Peptides (26th EPS) aura lieu à Montpellier, France du 10 au 15 septembre 2000. C'est un événement biennal, qui regroupe plus d'un millier de personnes et qui est le congrès de référence dans le monde du Peptide (le dernier symposium, qui s'est déroulé en France, a été organisé par le Professeur Bricas en 1968). Il est organisé sous les auspices de la Société Européenne des Peptides (EPS) et, cette année, du Groupe Français des Peptides et Protéines (GFPP). L'organisateur, le Professeur Jean Martinez, vous attend à Montpellier.

- Un présymposium sur le suivi analytique des réactions organiques sur support solide aura lieu le samedi 9 septembre 2000 et est organisé par le Professeur Jean-Louis Aubagnac.

- Consultez notre site web pour toute information et inscription.

$$
\text { Site web : http//ww2.pharma.univ.montp1.fr/26-EPS }
$$

